\title{
STATISTICAL GAUSSIAN DISTRIBUTION FUNCTION AS A DISTANCE INDICATOR TO STELLAR GROUPS
}

\author{
H. I. Abdel-Rahman ${ }^{1,2}$, I. A. Issa ${ }^{1}$, M. A. $\operatorname{Sharaf}^{3}$, M. I. Nouh ${ }^{1,5}$, A. Bakry ${ }^{4}$, A. I. Osman ${ }^{4}$, \\ A. S. SAAD ${ }^{1}$, F. Y. KAMAL ${ }^{4}$, AND ESSAM ${ }^{1}$ \\ 1 National Research Institute of Astronomy and Geophysics, Helwan, Cairo, Egypt \\ 2 Department of Mathematics, Al-Aflag Community college, King Saud Univ., Riyadh, Saudi Arabia \\ 3 Department of Astronomy, Faculty of Science, King Abdul Aziz Univ., Jeddah, Saudi Arabia \\ ${ }^{4}$ Department of Astronomy, Faculty of Science Al-Azhar Univ., Cairo, Egypt \\ ${ }^{5}$ Department of Physics, Faculty of Science, Northern Borders Univ., Arar, Saudi Arabia \\ (Received January 8, 2009; Accepted August 7, 2009)
}

\begin{abstract}
In this paper, statistical distribution functions are developed for distance determination of stellar groups. This method depends on the assumption that absolute magnitudes and apparent magnitudes follow a Gaussian distribution function. Due to the limits of the integrands of the frequency function of apparent and absolute magnitudes, we introduce Case A, B, and C Gaussian distributions. The developed approaches have been implemented to determine distances to some clusters and stellar associations. The comparison with the distances derived by different authors reveals good agreement.
\end{abstract}

Key words : Distance scales; Methods: statistical; Galaxies: star clusters; Stars: fundamental parameters

\section{INTRODUCTION}

Trigonometric, spectroscopic, dynamical parallaxes (Jenkins,1952, Gliese, 1978, Mihalas and Binney 1981, Wilson and Bappu 1957; Blitz, 1980 ) are methods that can be used to determine distances to objects as far as some tens of parsec to some hundreds. Moving star clusters, zero age main sequence fitting are also two other methods for distance determination (Heck, 1978; Blaauw, 1973). Issa (1980, 1981, 1982, 1985) used the size distribution functions of dark clouds, HII- region radii and globular clusters for the distances determination.

Most important is the standard candle procedure (Sandage and Tammann, 1971, Gascoigne, 1974, Martin et al., 1979, Iben and Tuggle, 1975, Vaucouleurs, 1979, van den Bergh, 1979; Hartwick and Hutchings, 1978) used to estimate distances to nearby objects as well as for remote galaxies and clusters of galaxies. Required always are some other independent means to establish distances to heavenly bodies. This is to assume that already determined distances are reliable and to be used wherever other distance means cannot be used. The term Malmqvist bias has been used in many places to describe related but different effects (Willick 1991). In the classical sense, the term Malmquist bias refers to a positive bias in the luminosity of objects in magnitude limited samples, as originally described by Malmquist in 1922 (Malmquist 1922). However, the term is now also commonly used for a related geometric

Corresponding Author: H. I. Abdel-Rahman bias that arises in distance measurements (Lynden-Bell et al. 1988). Malmquist bias is a statistical effect and formally can be evaluated using probability theory. In this work we reanalyze Sharaf et. al. (2003) method with the aim to discuss some special cases which may be of importance. We treated Malmquist bias (Sharaf et. al 2007) although of being less important in clusters of stars. But we still believe that the data used in these clusters do not represent a complete sample (membership problem). Also selection effects are still important in these clusters. The organization of the paper is as follows. The method of calculation is discussed in Section 2. Section 3 is devoted to the data sources. Results and discussions are represented in Section 4.

\section{THE METHOD}

We assume that all the members in a given cosmic group are at the same distance, $r$ parsec. The frequency function of the absolute magnitudes of the members of a given cosmic group could be given by

$\Phi(M)=\frac{1}{\sigma \sqrt{2 \pi}} e^{-\left(M-M_{0}\right)^{2} / 2 \sigma^{2}} .(-\infty \leq M \leq \infty)$,

showing that the members are scattered around a mean absolute magnitude, in normal distribution with dispersion. It should be noted that the dispersion arises from uncertainties in some factors, e.g., spectral classification and the actual cosmic dispersion in absolute magnitude which is due to evolution.

The relation between the apparent magnitude $m$ and 
the absolute magnitude $M$ of a cosmic object at a distance $r$ (in parsecs) is given by

$$
M=m+5-5 \log r .
$$

The frequency function of the apparent magnitudes is written as

$$
\Psi(m)=\frac{\partial(r, m)}{\partial(r, M)} \Phi[M(m, r)]
$$

where $r$ is assumed to be constant for all the members of the group. From Equations (1), and (2) in (3) we get

$$
\Psi(m)=\frac{1}{\sigma \sqrt{2 \pi}} e^{-\left(m+5-\log r-M_{0}\right)^{2} / 2 \sigma^{2}} .
$$

Let us consider all member brighter than a given apparent magnitude $m_{L}$ (a certain limiting magnitude). Then the mean magnitude is given by

$$
\bar{m}=\frac{\int_{-\infty}^{m_{1}} m \Psi(m) d m}{\int_{-\infty}^{m_{1}} \Psi(m) d m} .
$$

Using equation (4) we obtain

$$
\bar{m}=\frac{\int_{-\infty}^{m_{1}} m e^{-\left(m+5-\log r-M_{0}\right)^{2} / 2 \sigma^{2}} d m}{\int_{-\infty}^{m_{1}} e^{-\left(m+5-\log r-M_{0}\right)^{2} / 2 \sigma^{2}} d m} .
$$

The distance $r$ could be determined from

$$
r=10^{\left(1+m_{L}-m_{0}-\sigma y\right) / 5}
$$

where $y$ is the solution of the transcendental equation

$$
\begin{gathered}
G(y)=y+e^{-\frac{y^{2}}{2}}\left\{\sqrt{\frac{\pi}{2}}\left[1+\operatorname{erf}\left(\frac{y}{\sqrt{2}}\right)\right]\right\}^{-1}-\alpha=0 \\
\alpha=\frac{m_{L}-\bar{m}}{\sigma}
\end{gathered}
$$

and $\operatorname{erf}(x)$ is the error function defined by the series

$$
\operatorname{erf}(x)=\frac{2}{\sqrt{\pi}} \sum_{n=0}^{\infty} \frac{(-1)^{n}}{n !(2 n+1)} z^{2 n+1} .
$$

If $A$ is the interstellar absorption coefficient and $[\mathrm{Fe} / \mathrm{H}]$ is the metallicity, then Equation (7) should be written as:

$$
r=10^{1+\left(m_{L}-M_{0}-\sigma y-A-[\mathrm{Fe} / \mathrm{H}]\right) / 5} .
$$

The distance modulus is then given by

$$
m-M=m_{L}-M_{0}-\sigma y-A-[\mathrm{Fe} / \mathrm{H}] .
$$

From the features of Equation (5), we could indicate that the limits of the integration represent the magnitude limit of the sample under investigations. So, by changing these limits, we could obtain different cases. Doing that, we obtained two additional cases, which are called Gaussian B (GB) and Gaussian C (GC). GB is a result of considering the data sample having two extremes $m_{g}$ (the brightest one) and $m_{L}$ (the faintest one). GC is raised as a result of taking the Sun as the brightest object $\left(m_{L}\right)$ and $m_{g}$ is minus infinity.

\section{(a) Case A (GA)}

The approach introduced by Sharaf et al. (2003) has been followed. Sharaf et al. (2003) adopted the mean absolute magnitude $\left(M_{0}\right)$ and dispersion given by Mihalas and Binney (1981). Sharaf et al. (2007) derived the dispersion corresponding to the minimum percentage error. These provide the mean absolute magnitudes for each spectral subtype and the dispersion as well. This way was followed instead of the one used earlier which depends on evolutionary theories which are not well understood (Mihalas and Binney, 1981. Malmquist Bias formula was used to get the best mean absolute magnitudes. We calculated for the cluster as a unit, for each spectral type in the cluster and whenever possible for spectral subtypes.

\section{(b) Case B (GB)}

Sharaf et al. (2003) assumed that all the members in a given cosmic group are nearly at the same distance, $r$ (in parsecs) and are normally distributed. Statistically, this can be written as $M \approx N\left(M ; M_{0}, \sigma_{M}^{2}\right)$. In the present work we add also the assumption of normality of $m \approx N\left(m ; \bar{m}_{0}, \sigma_{m}^{2}\right)$. That is both the absolute $(8)$ and apparent magnitudes are assumed to be normally distributed.

In the present case (GB), we changed the limits of the integrands from that used by Sharaf et al. (2003) in such way that we considered all members between two apparent magnitudes $m_{L}$ and $m_{g}\left(m_{L}>m_{g}\right)$ where $m_{L}$ is the faintest side and $m_{g}$ is the brightest side of the star cluster sample. Changing the limits of integration requires adding another variable to the problem, which facilitates the optimization process and may lead to better results. This resulted also in adding two error functions instead of one. The mean apparent magnitude is given as

$$
\bar{m}_{B}=\frac{\int_{m_{g}}^{m_{L}} m \Psi(m) d m}{\int_{m_{g}}^{m_{L}} \Psi(m) d m} .
$$


Substituting Eqs. (4) and (5)we obtain

$$
\bar{m}_{B}=\frac{\int_{m_{g}}^{m_{L}} m e^{-\left(m+5-\log r-M_{0}\right)^{2} / 2 \sigma^{2}} d m}{\int_{m_{g}}^{m_{L}} e^{-\left(m+5-\log r-M_{0}\right)^{2} / 2 \sigma^{2}} d m}
$$

It can be shown that, the distance $r$ could be determined from

$$
r_{B}=10^{\left(1+m_{L}-M_{0}-\sigma y_{B}\right) / 5},
$$

where $y$ is the solution of the transcendental equation

$$
\begin{gathered}
G\left(y_{B}\right)=y_{B}-\left\{e^{-\frac{z_{1}^{2}}{2}}+e^{-z_{1} y_{B}}\right\} \\
\left\{\sqrt{\frac{\pi}{2}}\left[\operatorname{erf}\left(\frac{y_{B}}{\sqrt{2}}\right)+\operatorname{erf}\left(\frac{z_{B}}{\sqrt{2}}\right)\right]\right\}^{-1}-\alpha_{B}=0
\end{gathered}
$$

and

$$
\begin{array}{r}
\alpha_{B}=\frac{m_{L}-\bar{m}_{B}}{\sigma}, z_{B}=z_{1}+y_{B} \\
\text { and } \quad z_{1}=\frac{m_{g}-m_{L}}{\sigma} .
\end{array}
$$

The general definition of the error function is

$$
\operatorname{erf}(x)=\frac{2}{\sqrt{\pi}} \int_{0}^{x} e^{-\frac{x^{2}}{2}} d x
$$

Clearly, $y$ is a basic quantity for distance determination of a given stellar group. The interstellar absorption $A$ and metallicity $[\mathrm{Fe} / \mathrm{H}]$ as well can be taken into account, then Equation (15) should be written as:

$$
r_{B}=10^{1+\left(m_{L}-m_{0}-\sigma y_{B}-A[F e / H]\right) / 5},
$$

The distance modulus is then given by

$$
(m-M)_{B}=m_{L}-M_{0}-\sigma y_{B}-A-[F e / H] .
$$

Increasing $[\mathrm{Fe} / \mathrm{H}]$ values affects the luminosity negatively and hence the magnitudes and the distances will increase. The metallicity must be subtracted if positive and neglected when negative.

\section{(c) Case C (GC)}

Equations (1) to (4) are also the starting point of this section. Here, we introduce another limit of $m_{L}$ and $m_{g}$, and we still consider $m_{L}>m_{g}$. We shall fix the limiting magnitude at the faintest limit reached by the Hubble Space Telescope at $m_{L}=26$ magnitude, while the apparent magnitude of the Sun is $m_{g}=-26.8$ which is the brightest magnitude.

Then after some manipulations we get the distance $r$ as follows

$$
r_{B}=10^{1+\left(26-M_{0}-\sigma y_{C}\right) / 5}
$$

where $y_{C}$ is the solution of the transcendental equation

$$
\begin{gathered}
G\left(y_{C}\right)=y_{C}-\left\{e^{-\frac{z_{2}^{2}}{2}}+e^{-z_{2} y_{C}}\right\} \\
\left\{\sqrt{\frac{\pi}{2}}\left[\operatorname{erf}\left(\frac{y_{C}}{\sqrt{2}}\right)+\operatorname{erf}\left(\frac{z_{C}}{\sqrt{2}}\right)\right]\right\}^{-1}-\alpha_{C}=0,
\end{gathered}
$$

and

$$
\begin{aligned}
\alpha_{C}=\frac{26-\bar{m}_{B}}{\sigma}, z_{C} & =z_{2}+y_{C} \\
\text { and } \quad z_{2} & =-\frac{52.8}{\sigma} .
\end{aligned}
$$

The metallicity $[\mathrm{Fe} / \mathrm{H}]$ as well can be taken into account, and Equation (21) must be written as:

$$
r_{C}=10^{1+\left(26-M_{0}-\sigma y_{C}-A-[F e / H]\right) / 5} .
$$

The distance modulus is then given by

$$
(m-M)_{C}=26-M_{0}-\sigma y_{C}-A-[\mathrm{Fe} / H]
$$

\section{DATA SOURCES}

Two types of cosmic objects are used for the present application, open star clusters and stellar associations.

\section{(a) Open Star Clusters}

UBVRI photometric observations for NGC 2658 and NGC 2439 open star clusters (Ramsay and Pollaco 1992) were used. The observations were obtained in 1990 Jan. 9-22 with the 1 meter telescope in Sutherland station of the South African Astronomical Observatory (SAAO). Vazquez and Will (1995), and Vazquez and Baume (1996) used the 0.61 meter telescope of the university of Toronto Southern observatory at Las Campanas Observatory, Chile, to get photometric observations for the open clusters Pis 20 and Tr 14 respectively.

\section{(b) Stellar Associations}

Brown et. al. (1994) used the Dutch 0.91 meter telescope at ESO in the VBLUW Walraven system, to get photoelectric observation for Orion OB1 associations. The final HIPPARCOS Input Catalog contains 699 stars of the sample under study (1318 stars in Orion OB1) of which 236 are of priority 1, and 463 are of priority 2 . These stars are the subject of this 
TABLE 1.

GA PARAMETERS AND DISTANCES $r_{s}$ OF THE PRESENT WORK IN COMPARISON TO OTHERS $r_{c}$.

\begin{tabular}{|c|c|c|c|c|c|c|c|c|}
\hline Objects & $m_{l}$ & $\bar{m}$ & $\sigma$ & $M_{0}$ & $\alpha$ & $\mathrm{Y}$ & $r_{s}(\mathrm{pc})$ & $r_{c}(\mathrm{pc})$ \\
\hline NGC 2658 & 16.69 & 14.02 & 0.32 & 1.04 & 8.22 & 8.22 & 3907.91 & $3631^{1}$ \\
\hline NGC & \multirow[t]{2}{*}{16.57} & \multirow[t]{2}{*}{13.42} & \multirow[t]{2}{*}{0.31} & \multirow[t]{2}{*}{0.26} & \multirow[t]{2}{*}{10.06} & \multirow[t]{2}{*}{10.06} & \multirow[t]{2}{*}{4296.10} & 4450 \\
\hline 2439 & & & & & & & & $\begin{array}{c}\text { Ramsay } \\
(1992)\end{array}$ \\
\hline \multirow[t]{2}{*}{ Pis 20} & \multirow[t]{2}{*}{13.66} & \multirow[t]{2}{*}{11.48} & \multirow[t]{2}{*}{0.01} & \multirow[t]{2}{*}{-1.31} & \multirow[t]{2}{*}{217.10} & \multirow[t]{2}{*}{217.10} & \multirow[t]{2}{*}{3630.78} & 3600 \\
\hline & & & & & & & & $\begin{array}{c}\text { Vazquezy } \\
(1995)\end{array}$ \\
\hline \multirow[t]{2}{*}{$\operatorname{Tr} 14$} & \multirow[t]{2}{*}{17.22} & \multirow[t]{2}{*}{12.93} & \multirow[t]{2}{*}{0.015} & \multirow[t]{2}{*}{0.43} & \multirow[t]{2}{*}{285.93} & \multirow[t]{2}{*}{285.73} & \multirow[t]{2}{*}{3359.50} & 3100 \\
\hline & & & & & & & & $\begin{array}{c}\text { Vazquezy } \\
(1996)\end{array}$ \\
\hline Orion & \multirow[t]{3}{*}{10.72} & \multirow[t]{3}{*}{8.32} & \multirow[t]{3}{*}{0.013} & \multirow[t]{3}{*}{0.79} & \multirow[t]{3}{*}{184.43} & \multirow[t]{3}{*}{184.43} & \multirow[t]{3}{*}{319.90} & 380.189 \\
\hline OB1 & & & & & & & & Brown \\
\hline $1 a$ & & & & & & & & $\begin{array}{c}(1994) \\
363\end{array}$ \\
\hline Orion & \multirow[t]{2}{*}{10.96} & \multirow[t]{2}{*}{7.99} & \multirow[t]{2}{*}{0.035} & 0.49 & 84.81 & 84.81 & 315.50 & 363.078 \\
\hline $\begin{array}{c}\text { OB1 } \\
1 \mathrm{~b}\end{array}$ & & & & & & & & $\begin{array}{l}\text { Brown } \\
(1994)\end{array}$ \\
\hline Orion & 10.31 & 8.25 & 0.021 & 0.85 & 97.74 & 97.74 & 302.80 & 398.107 \\
\hline OB1 & & & & & & & & Brown \\
\hline $1 c$ & & & & & & & & (1994) \\
\hline Orion & 9.19 & 6.71 & 0.073 & -1.36 & 33.91 & 33.91 & 412.40 & 380.189 \\
\hline OB1 & & & & & & & & Brown \\
\hline $1 d$ & & & & & & & & (1994) \\
\hline Car & 9.60 & 6.98 & 0.014 & -4.85 & 187.11 & 187.11 & 2331.99 & 2850 \\
\hline OB1 & & & & & & & & $\begin{array}{c}\text { Kaltcheva } \\
\text { (1998) }\end{array}$ \\
\hline Car & 9.20 & 7.45 & 0.015 & -4.20 & 116.66 & 116.66 & 2149.01 & 2590 \\
\hline OB2 & & & & & & & & $\begin{array}{c}\text { Kaltcheva } \\
\text { (1998) }\end{array}$ \\
\hline
\end{tabular}

${ }^{1}$ Tadross (2002), ${ }^{2}$ Ramsey (1992),

work. Kaltcheva (1998) observed Carina OB1 and Carina OB2 using the half meter telescope at Sutherland site of the SAAO. The observations were calibrated to the standard system using standard stars from the lists of Cousins (1987), Perry et al. (1987), Crawford et al. (1971) and Olsen (1983). The reduction to the standard system was carried out by the procedure adopted at the SAAO.

To correct the apparent magnitude for absorption we use the relation

where

$$
m_{0}=m-A_{v}
$$

$$
A_{v}=3.1 E(B-V)
$$

and $A_{v}, B, V$ and $E(B-V)$ are the visual absorption, the magnitudes in the blue and visual filters, and the color excess respectively.

\section{NUMERICAL RESULTS AND DISCUS- SIONS}

All computations are carried out through computational algorithms which were coded using Mathematica software. This was applied for all approaches mentioned in Section 2.

\section{(a) Case A (GA)}

The result of Case A is presented in Table (1), which has the following designations: column 1 stands for the object name, column 2 is a certain limiting magnitude $m_{L}$, column 3 is the mean apparent magnitude $\bar{m}$, column 4 is the dispersion $\sigma$, column 5 is the mean absolute magnitude $M_{0}$, column 6 contains the parameter $\alpha$ and column 7 gives $y$ the solution of the transcendental equation. Columns 8 and 9 give the statistical distance computed by the present approach and $r_{s}$ is the distance computed by others. In comparison to other methods we believe that our method is most accept- 
TABLE 2.

GB PARAMETERS AND DISTANCES $r_{s}$ OF THE PRESENT WORK IN COMPARISON TO OTHERS $r_{c}$.

\begin{tabular}{|c|c|c|c|c|c|c|c|c|c|}
\hline Objects & $m_{l}$ & $m_{g}$ & $\bar{m}$ & $\sigma$ & $M_{0}$ & $\alpha$ & $Y$ & $r_{s}(\mathrm{pc})$ & $r_{c}(\mathrm{pc})$ \\
\hline $\begin{array}{l}\text { NGC } \\
2658\end{array}$ & 16.69 & 11.88 & 14.02 & 1.31 & 3.28 & 2.04 & 0.18 & 4278.20 & $\begin{array}{c}3631 \\
\text { Tadross } \\
(2002)\end{array}$ \\
\hline $\begin{array}{c}\text { NGC } \\
2439\end{array}$ & 16.57 & 7.60 & 13.42 & 1.441 & 3.03 & 2.16 & 0.13 & 4668.90 & $\begin{array}{c}4450 \\
\text { Ramsay } \\
(1992)\end{array}$ \\
\hline Pis 20 & 13.66 & 4.45 & 11.48 & 1.22 & 0.74 & 1.77 & 0.09 & 3631.40 & $\begin{array}{c}3600 \\
\text { Vazquezy } \\
(1995)\end{array}$ \\
\hline $\operatorname{Tr} 14$ & 17.22 & 4.97 & 12.93 & 1.71 & 4.49 & 2.50 & 0.13 & 3156.70 & $\begin{array}{c}3100 \\
\text { Vazquezy } \\
(1996)\end{array}$ \\
\hline $\begin{array}{l}\text { Orion } \\
\text { OB1 } \\
1 \mathrm{a}\end{array}$ & 10.72 & 3.91 & 8.32 & 1.22 & 2.86 & 1.96 & 0.12 & 346.87 & $\begin{array}{l}380.18 \\
\text { Brown } \\
(1994)\end{array}$ \\
\hline $\begin{array}{l}\text { Orion } \\
\text { OB1 } \\
\text { 1b }\end{array}$ & 10.96 & 1.66 & 7.99 & 1.38 & 3.13 & 2.14 & 0.12 & 339.07 & $\begin{array}{c}363.078 \\
\text { Brown } \\
(1994)\end{array}$ \\
\hline $\begin{array}{l}\text { Orion } \\
\text { OB1 } \\
1 \mathrm{c}\end{array}$ & 10.31 & 2.07 & 8.25 & 1.12 & 2.59 & 1.82 & 0.09 & 332.58 & $\begin{array}{l}398.10 \\
\text { Brown } \\
(1994)\end{array}$ \\
\hline $\begin{array}{l}\text { Orion } \\
\text { OB1 } \\
\text { 1d }\end{array}$ & 9.19 & 4.10 & 6.71 & 1.18 & 0.58 & 2.08 & 0.17 & 479.48 & $\begin{array}{l}380.18 \\
\text { Brown } \\
(1994)\end{array}$ \\
\hline $\begin{array}{c}\text { Car } \\
\text { OB1 }\end{array}$ & 9.60 & 4.17 & 6.98 & 1.18 & -2.91 & 2.21 & 0.17 & 2911.30 & $\begin{array}{c}2850 \\
\text { Kaltcheva } \\
(1998)\end{array}$ \\
\hline $\begin{array}{c}\text { Car } \\
\text { OB2 }\end{array}$ & 9.20 & 4.88 & 7.45 & 0.93 & -2.98 & 1.86 & 0.14 & 2582.60 & $\begin{array}{c}2590 \\
\text { Kaltcheva } \\
(1998)\end{array}$ \\
\hline
\end{tabular}

able. Although standard candles were studied and understood well, we believe that some evolutionary facts (exact position in H-R Diagram, Hayashi tracks, instability strip width for Cepheids, the low mass tail at the end of the main sequence) still need more understanding. Interstellar extinction values are still and perhaps will remain one of the most dangerous difficulties for distance determination. Accordingly, distances derived from different photometric methods for the same object still show significant differences. Our approach is unique, using the known facts of statistics for well defined parameters of a distribution function which has a universal nature.

\section{(b) Case B (GB)}

In Table (2): column 1 is devoted for the object name, column 2 for the limiting magnitude $m_{L}$, column 3 for $m_{g}$, and the other columns have the same designation as Table 2. Our distances $r_{s}$ differ a bit from the distances $r_{c}$ obtained by other methods. Our distances for NGC 2658, NGC 2439, Pis 20, Tr 14, Car OB1 and Orion OB1-1d are little big while in some others are bit smaller. One must mention that $r_{c}$ obtained mostly through photoelectric techniques suffering from many approximations especially the extinction, either on the way of the light to us or the extinction of light through the group itself and suffers from some evolutionary factors still remaining to affect the distances. While the statistical methods smears all these factors, and remains of statistical nature. We would like to remind the reader to the fact that dividing the HIPPARCOS stars into priority 1 and priority 2 stars may throw some doubt about the accuracy of their distances.

\section{(c) Case C (GC)}

In Table (3), the results of this approach are given. Columns 1 to 6 are the object name, the mean apparent magnitude $\bar{m}$, the dispersion $\sigma$, the mean absolute 
TABLE 3.

GC PARAMETERS AND DISTANCES $r_{s}$ OF THE PRESENT WORK IN COMPARISON TO OTHERS $r_{c}$.

\begin{tabular}{|c|c|c|c|c|c|c|c|}
\hline Objects & $\bar{m}$ & $\sigma$ & $M_{0}$ & $\alpha$ & $\mathrm{Y}$ & $r_{s}(\mathrm{pc})$ & $r_{c}(\mathrm{pc})$ \\
\hline $\begin{array}{l}\text { NGC } \\
2658\end{array}$ & 14.02 & 2.91 & 12.59 & 4.12 & 0.085 & 4273.37 & $\begin{array}{c}3631 \\
\text { Tadross } \\
(2002)\end{array}$ \\
\hline $\begin{array}{l}\text { NGC } \\
2439\end{array}$ & 13.42 & 2.97 & 12.38 & 4.22 & 0.088 & 4678.23 & $\begin{array}{c}\text { Ramsay } \\
(1992)\end{array}$ \\
\hline Pis 20 & 11.48 & 3.20 & 12.88 & 4.52 & 0.099 & 3626.81 & $\begin{array}{c}3600 \\
\text { Vazquezy } \\
(1995)\end{array}$ \\
\hline $\operatorname{Tr} 14$ & 12.93 & 3.04 & 13.22 & 4.29 & 0.091 & 3158.93 & $\begin{array}{c}3100 \\
\text { Vazquezy } \\
(1996)\end{array}$ \\
\hline $\begin{array}{l}\text { Orion } \\
\text { OB1 } \\
\text { la }\end{array}$ & 8.32 & 3.51 & 17.89 & 5.02 & 0.114 & 347.58 & $\begin{array}{c}380.189 \\
\text { Brown } \\
(1994)\end{array}$ \\
\hline $\begin{array}{c}\text { Orion } \\
\text { OB1 } \\
\text { 1b }\end{array}$ & 7.99 & 3.55 & 17.93 & 5.06 & 0.116 & 339.72 & $\begin{array}{c}363.078 \\
\text { Brown } \\
(1994)\end{array}$ \\
\hline $\begin{array}{c}\text { Orion } \\
\text { OB1 } \\
\text { 1c }\end{array}$ & 8.25 & 3.52 & 17.98 & 5.03 & 0.114 & 332.80 & $\begin{array}{c}398.107 \\
\text { Brown } \\
(1994)\end{array}$ \\
\hline $\begin{array}{l}\text { Orion } \\
\text { OB1 } \\
\text { 1d }\end{array}$ & 6.71 & 3.66 & 17.15 & 5.26 & 0.122 & 478.62 & $\begin{array}{l}380.18 \\
\text { Brown } \\
(1994)\end{array}$ \\
\hline $\begin{array}{c}\text { Car } \\
\text { OB1 }\end{array}$ & 6.98 & 3.61 & 13.24 & 5.25 & 0.120 & 2904.72 & $\begin{array}{c}2850 \\
\text { Kaltcheva } \\
(1998)\end{array}$ \\
\hline $\begin{array}{c}\text { Car } \\
\text { OB2 }\end{array}$ & 7.45 & 3.58 & 13.51 & 5.17 & 0.118 & 2578.37 & $\begin{array}{c}2590 \\
\text { Kaltcheva } \\
(1998)\end{array}$ \\
\hline
\end{tabular}

magnitude $M_{0}$, the parameter $\alpha$ and the distance parameter $y$ is the solution of Equation (17) respectively. Columns 7 to 9 include $r_{s}$ the statistical distance computed by the present method and $r_{c}$ is the distance computed by the others and the references of comparisons respectively. Comparing our distances with those obtained by others still, indicates that our distances for NGC 2658, NGC 2439, Pis 20, Tr 14, Car OB1 and Orion OB1-1d are little bit bigger. Whereas, our distances for Orion OB1 (1a, 1b, 1c) and Car OB2 are smaller. This can be interpreted as due to uncertainties in estimating interstellar absorption in the other methods and we must not forget that our method is statistical, depending on the averages.

\section{CONCLUSION}

The main aim of this work was to put the theoretical statistical foundations of the distribution functions for cosmological distance determinations. The results of the present work are summarized in Table (4). The entries are $r_{s}$ : the distances reached in this work, where the subscript $s$ stands for statistical distances determined using statistical distribution function. $r_{c}$ distances from other methods, the subscript $c$ stands for comparison. Distances given in columns 3 and 4 differ slightly among each other. They differ a bit, however, from distances of column 2 named GA. The differences among distances in column 3, 4 increase as the distance increase, while they are small for small distances. Figure 1 plots the comparison between the statistical distance and the distance in the first row of the fifth column in Table 4. More samples are needed to establish the effect of changing $m_{L}$ and $m a_{g}$ on distances. No systematic difference are shown among our distances and the distance $r_{c}$. However it should be mentioned that, different author's distance do not agree fully among themselves. For NGC 2658 quite a big difference between distances given by Tadross (2002) and Loktin (2001). The same also for NGC 2439. Differ- 


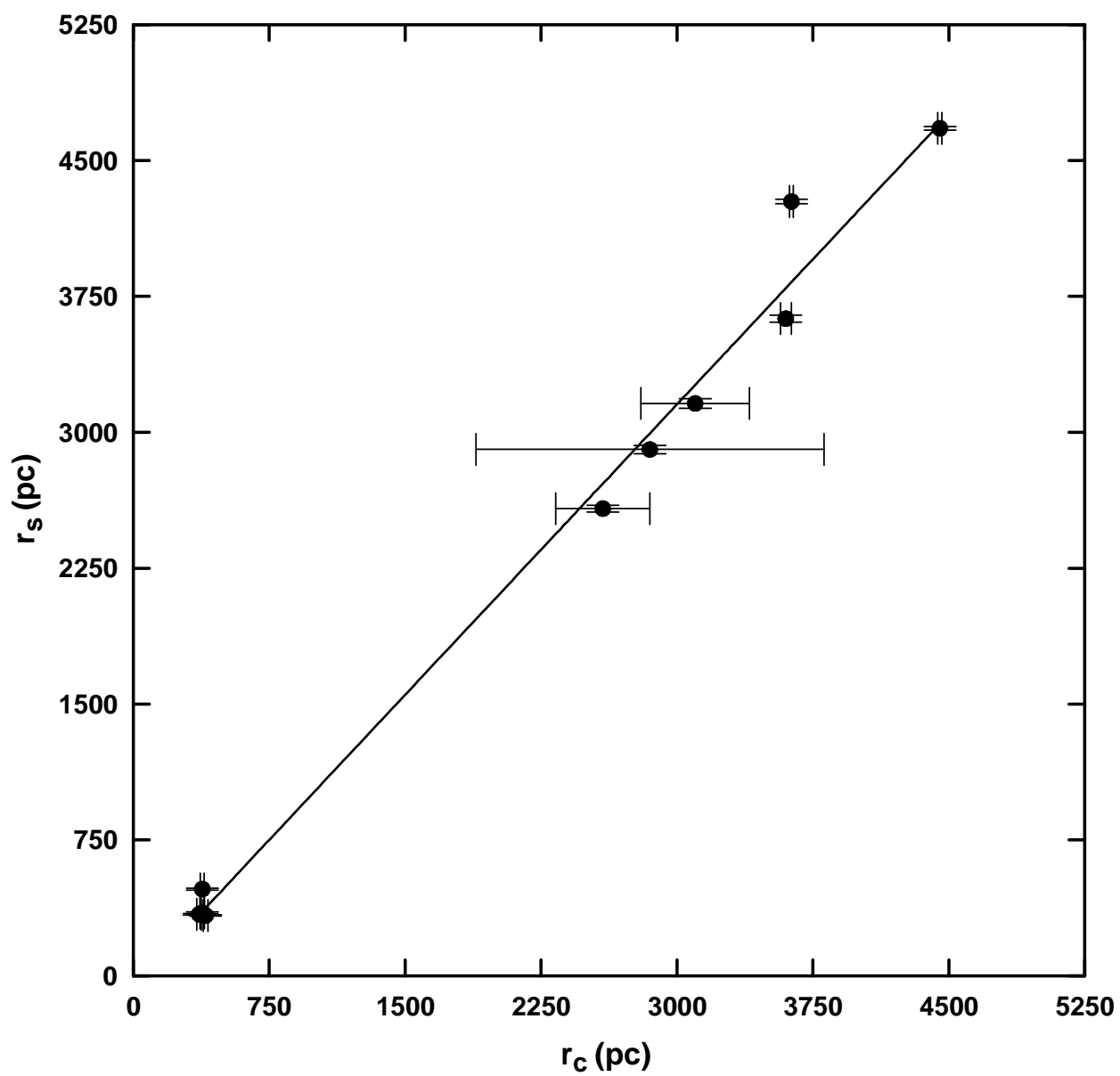

Fig. 1. - Comparison between the distance computed in the present work $\left(r_{s}\right)$ with the distance $\left(r_{c}\right)$ in the first row of the fifth column in Table 4.

ences also among other author's distances are found in Orion OB1 (1a, 1b, 1c). In our opinion such differences can be due to the location of the stars used to determine the distances. It can be on the near side or in the far side of the association. The difference among these distances is as big as the difference between the distances of the present work and the other distances, which is of the same order as the linear dimensions of the complex. The distance of the far side of the Orion OB1 is $500 \mathrm{pc}$ while the distance of the near side is as big as 300 pc. Depending on statistical distribution functions, although based on averages, and on observations taken by different authors, can give smearing out of some defects that are inherited in others distances. The smearing out means that stars from the far side are used in the distribution to determine the distance as well as stars of the near side. They were used with the same weight. The statistical methods smears all these factors, and remains of statistical nature. It is to be mentioned also that sub-grouping especially in Orion, and the position of the stars in the cluster or association used to determine the distance, affect them significantly. Since, the linear dimension of this structure is on average $45 \mathrm{pc}$ reaching sometimes to $100 \mathrm{pc}$. Interstellar absorption and the assumption regarding the ratio of total to selective absorption, produce also its uncertainties. We still like to remind the reader of the fact of dividing stars by some authors into priority 1 and priority 2 stars and its effect on distance determinations.

\section{REFERENCES}

van den Berg, S., 1977, The Distance Scale Within the Local Group, IAU colloq., 37, 13

Blaauw, A., 1973, The Calibration of Luminosity Criteria (survey Lecture), IAU Symp., 54, 47 
TABLE 4.

GC PARAMETERS AND DISTANCES $r_{s}$ OF THE PRESENT WORK IN COMPARISON TO OTHERS $r_{c}$.

\begin{tabular}{|c|c|c|c|c|c|}
\hline Objects & $r_{s}(\mathrm{pc}) \mathrm{GA}$ & $r_{s}(\mathrm{pc}) \mathrm{GB}$ & $r_{s}(\mathrm{pc}) \mathrm{GC}$ & $r_{c}(\mathrm{pc})$ & Ref. of $r_{c}$ \\
\hline NGC & \multirow{2}{*}{$3907.91 \pm 20$} & \multirow{2}{*}{$4278.20 \pm 10$} & \multirow{2}{*}{$4273.37 \pm 12$} & $3631.91 \pm 11$ & Tadross $(2002)$ \\
\hline 2658 & & & & 2021 & Loktin(2001) \\
\hline NGC & \multirow{2}{*}{$4293.10 \pm 17$} & \multirow[t]{2}{*}{$4668.90 \pm 9$} & \multirow[t]{2}{*}{$4678.23 \pm 10$} & $4450 \pm 11$ & Ramsay(1992) \\
\hline 2439 & & & & 3855 & Loktin (2001) \\
\hline \multirow[t]{2}{*}{ Psi 20} & \multirow{2}{*}{$3630.78 \pm 25$} & \multirow{2}{*}{$3631.40 \pm 16$} & \multirow{2}{*}{$3626.81 \pm 19$} & $3600 \pm 3$ & Vazquez (1995) \\
\hline & & & & 2018 & Loktin $(2001)$ \\
\hline \multirow[t]{2}{*}{$\operatorname{Tr} 14$} & \multirow[t]{2}{*}{$3159.50 \pm 32$} & \multirow[t]{2}{*}{$3156.70 \pm 24$} & \multirow[t]{2}{*}{$3158.93 \pm 27$} & $3100 \pm 300$ & Vazquez (1996) \\
\hline & & & & 2733 & Loktin(2001) \\
\hline Orion & \multirow[t]{2}{*}{$319.90 \pm 8$} & \multirow[t]{2}{*}{$346.87 \pm 4$} & \multirow[t]{2}{*}{$347.58 \pm 5.7$} & $380.18 \pm 13$ & Brown (1994) \\
\hline OB1-1a & & & & $398 \pm 12$ & Warren \& Hesser $(1978$ \\
\hline Orion & \multirow[t]{2}{*}{$315.50 \pm 7$} & \multirow[t]{2}{*}{$339.07 \pm 4$} & \multirow[t]{2}{*}{$3339.72 \pm 5$} & $363.07 \pm 12$ & Brown (1994) \\
\hline OB1-1b & & & & $443 \pm 12$ & Warren \& Hesser $(1978$ \\
\hline Orion & \multirow[t]{2}{*}{$302.80 \pm 7$} & \multirow[t]{2}{*}{$332.58 \pm 3$} & \multirow[t]{2}{*}{$332.80 \pm 4$} & $398.10 \pm 13$ & Brown (1994) \\
\hline OB1-1c & & & & $437 \pm 13$ & Warren \& Hesser $(1978$ \\
\hline Orion & \multirow[t]{2}{*}{$412.40 \pm 9$} & \multirow[t]{2}{*}{$479.48 \pm 4$} & \multirow[t]{2}{*}{$478.62 \pm 6$} & $380.18 \pm 11$ & Brown (1994) \\
\hline OB1-1d & & & & $479 \pm 13$ & Warren \& Hesser $(1978$ \\
\hline Car OB1 & $2331.99 \pm 24$ & $2911.30 \pm 22$ & $2904.72 \pm 23$ & $2850 \pm 961$ & Kaltcheva (1998) \\
\hline Car OB2 & $2149.01 \pm 22$ & $2582.60 \pm 14$ & $2578.37 \pm 18$ & $2590 \pm 260$ & Kaltcheva (1998) \\
\hline
\end{tabular}

Blitz, L., 1979, The rotation curve of the Galaxy to $\mathrm{R}$ = 16 kiloparsecs, ApJ, 231, L115

Brown, A. G. A., Degeus, E. J., \& Gezeeuw, P. T., 1994, The Orion OB1 association. 1: Stellar content, A\&A, 289, 101

Cousins, A. W. J., 1987, Secondary standards for the Stromgren UVBY system, SAAO Circ., 11, 93

Crawford D. L., Barnes J. V., \& Golson J. C., 1971, Four-color and Hbeta photometry for bright B-type stars in the southern hemisphere, AJ, 76, 621

Gascoigne, S. C. B., 1974, Metal abundance and the luminosities of cepheids, MNRAS, 166, 25

Gliese, W., 1978, Hertzsprung-Russell diagrams and color-luminosity diagrams for the stars nearer than twenty-two parsecs in The HR diagram - The 100th anniversary of Henry Norris Russell; Proceedings of the IAU Symposium 80, Washington, D.C., November 2-5, 1977. (A79-14326 03-89) Dordrecht, D. Reidel Publishing Co.

Hartwick, F. D. A. \& Hutchings, J. B., 1978, Classical novae - A time-dependent optically thick wind model for the postmaximum phase, ApJ, 226, 203

Heck, A., 1978, Some methods of determining the stellar absolute magnitude, vistas in Astron., 22, 221

Iben, I., Jr. \& Tuggle, R, S., 1975, On the intrinsic properties of cepheids in the galaxy, in Andromeda, and in the Magellanic Clouds, ApJ, 197, 39

Issa, I. A., 1980, On the dust content of nearby galaxies, $\mathrm{AN}, 301,177$
Issa, I. A., 1981, The size distribution of HII regions as a new variant to determine the distances of galaxies, AN, 302, 251.

Issa, I. A., 1982, The amount of dust and the distance of NGC 253, AN, 303, 127

Issa, I. A., 1985, Distance estimate of M31 based on the size distribution of H II regions and dark clouds, Astrophysics and Space Science, 113, 317

Jenkins, L. F., 1952, General catalogue of trigonometric stellar parallaxes, Yale university press

Kaltcheva, N. T., 1998, Stromgren and H photometry of $\mathrm{OB}$ stars in the region of the Carina Spiral Feature, A\&AS, 128, 309

Loktin, A. V., Gerasimenko, T. P., \& Malisheva, L. K., 2001, The catalogue of open cluster parameterssecond version, A\&AT, 20, 607

Lynden-Bell, D., Faber, S. M., Burstein, David, Davies, Roger L., Dressler, Alan, Terlevich, R. J.,\& Wegner, Gary, 1988, Spectroscopy and photometry of elliptical galaxies. V - Galaxy streaming toward the new supergalactic center, ApJ, 326, 19

Malmquist, K. G., 1922, Jour. Medd. Lund Astron. Obs. Ser. II, 32, 64

Martin, W. L., Warren, P. R., \& Feast, M. W., 1979, Multicolour photoelectric photometry of Magellanic Cloud Cepheids. II - an analysis of BVI observations in the LMC, MNRAS, 188, 139

Mihalas, D. \& Binney, J., 1981, Galactic Astronomy, 2nd Ed. Freeman, San Francisco 
Perry C. L., Olsen E. H., \& Crawford D. L., 1987, A catalog of bright UVBY beta standard stars, PASP, 99, 1184

Ramsay, G. \& Pollaco, D. L., 1992, CCD observations in 7 open clusters - NGC2421, NGC2439, NGC2489, NGC2567, NGC2627, NGC2658 and NGC2910, A\&AS, 94, 73

Sandage, A. \& Tammann, G. A., 1971, Absolute Magnitudes of Cepheids. III. Amplitude as a Function of Position in the Instability Strip: a PeriodLuminosity Relation, ApJ, 167, 293

Sharaf, M. A., Issa, I. A., \& Saad, A. S., 2003, A method for the determination of cosmic distances, New Astronomy, 8, 15

Sharaf, M. A., Abdel-Rahman, Helal I., Nouh, M. I., Saad, A. S., Osman, A. I., Ahmed, A. B., Mohanna, M., \& Issa, I. A., 2007, Journal of the Astronomical Society of Egypt, Vol. 14/ii, pp24-33.

Tadross, A. L., Werner, P., Osman, A., \& Marie, M., 2002, Morphological analysis of open clusters' propertiesII. Relationships projected onto the galactic plane, New Astronomy, 7, 553

Vaucoulers, G., de, 1979, The extragalactic distance scale. VI - Distances of 458 spiral galaxies from tertiary indicators, ApJ, 227, 729

Vazquez, R. A. \& Will, J. M., 1995, A detailed study of the open cluster PISMIS 20, A\&AS, 111, 85

Vazque, R. A. \& Baume, G., 1996, Investigation on the region of the open cluster TR 14, A\&AS, 116, 75

Willick, J. 1991, Calibration of the CCD Tully-Fisher relation with application to the Perseus-Pisces supercluster, PhD. thesis, University of California, Berkeley

Wilson, O. C. \& Bappu, M. K. V., 1957, H and K Emission in Late-Type Stars: Dependence of Line Width on Luminosity and Related Topics, ApJ, 125, 661 